\title{
Torsional Stiffness Calculation of Double-Enveloping Cycloid Drive*
}

\author{
Jingya LIU**, Shigeki MATSUMURA***, Bingkui CHEN** \\ and Haruo $\mathrm{HOUJOH}^{* * *}$ \\ ${ }^{* *}$ The State Key Laboratory of Mechanical Transmission, Chongqing University \\ 174 Shazheng Street, Shapingba, Chongqing, China \\ E-mail: jyliu_china@yahoo.com.cn \\ ***Precision and Intelligence Laboratory, Tokyo Institute of Technology \\ 4259 Nagatsuta, Midori-ku, Yokohama, Japan
}

\begin{abstract}
On a gear tooth surface, there may be some certain points which contact twice with its mating gear tooth surface. This phenomenon is called secondary action. In this paper, applying this phenomenon in cycloid drive, a new type of double-enveloping cycloid drive is generated based on gear geometry theory, and the torsional stiffness is calculated. The new conjugated tooth profile is composed by an external cycloid tooth profile and a new generated internal ring gear tooth profile. The main characteristic of the new tooth profile is that there are double contact lines simultaneously between on tooth pair in a certain meshing area. In this paper, firstly the generation method of the new conjugated tooth profile is present, and the meshing characteristics are investigated. Secondly, the torsional stiffness calculation model for double-enveloping cycloid drive is established. The normal stiffness, the arm length of the meshing force and the torsional stiffness are calculated. Finally, based on a numerical example, the calculation results are present, and the torsional stiffness of double-enveloping cycloid drive is compared with that of the conventional cycloid drive both on single tooth pair and whole gear mesh.
\end{abstract}

Key words: Cycloid Drive, Double-Enveloping, Double Contact Lines, Normal Stiffness, Arm Length, Torsional Stiffness

\section{Introduction}

Cycloid drive has been already used widely in many industrial areas for power transmission because of its large transmission ratio, compact size, large load capacity and high efficiency. In addition the characteristic of multi-tooth meshing simultaneously can lead to high transmission precision because of the averaging effect of errors. Also compared with strain wave gear reducer, there is no flexible element in cycloid drive which can lead to high stiffness. So in recent years, cycloid drive is also used commonly in precision transmission, such as robot and aerospace area ${ }^{(1)}$.

On a gear tooth surface there may be some certain points which contact twice with its mating gear tooth surface ${ }^{(2)}$. This phenomenon which is called secondary action has been investigated and researched in skew gears, especially in worm gears by many researchers $^{(3)(4)}$. However the secondary action of cycloid drive is studied very little. Applying this secondary action phenomenon in cycloid drive, a new conjugated tooth profile with double contact lines between one tooth pair is present.

The variation of tooth meshing stiffness is one of the main causes of the gear pair

${ }^{*}$ Received 1 Aug., 2011 (No. 11-0491) [DOI: 10.1299/jamdsm.6.2]

Copyright $@ 2012$ by JSME 
vibration. So the tooth meshing stiffness is one important parameter to evaluate the dynamic behavior of a gear pair ${ }^{(5)}$. The stiffness of conventional cycloid drive has been calculated by some researchers ${ }^{(6)}$. However, nearly no researchers focus on the stiffness calculation of the double-enveloping cycloid drive.

In this paper, firstly based on gear geometry theory the meshing equation and the equation of the new tooth profile are present, the meshing characteristic of double contact is investigated. Secondly, the stiffness calculation model for the double-enveloping cycloid drive is established. The normal stiffness, the arm length and the torsional stiffness are calculated. Finally, based on a numerical example, the calculation results are present, and the torsional stiffness of the double-enveloping cycloid drive is compared with that of the conventional cycloid drive.

\section{Double-enveloping cycloid drive}

\subsection{Generation of new conjugated tooth profile}

For the conventional cycloid drive, the conjugated meshing gear pair is composed by an external cycloid gear and the cylindrical pin rollers, as Fig. 1. Applying secondary action phenomenon in cycloid drive, a new conjugated gear pair can be generated using double-enveloping method ${ }^{(7)}$. The new conjugated gear pair is composed by an external cycloid gear and an new internal ring gear, as Fig. 2. The main characteristic of the new conjugated tooth profile is that there are double contact lines between one tooth pair in a certain meshing area.

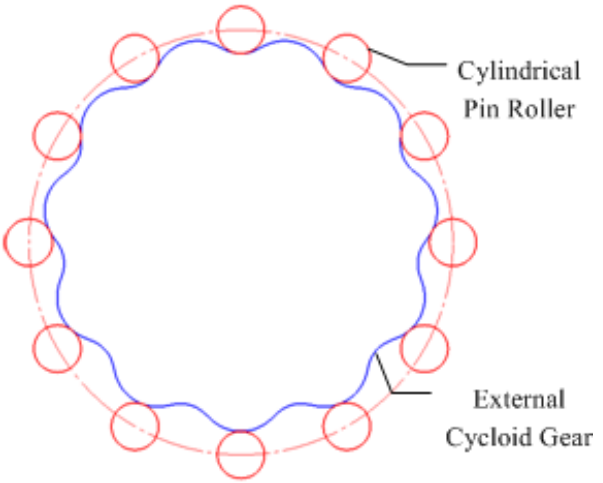

Fig. 1 Conventional cycloid drive

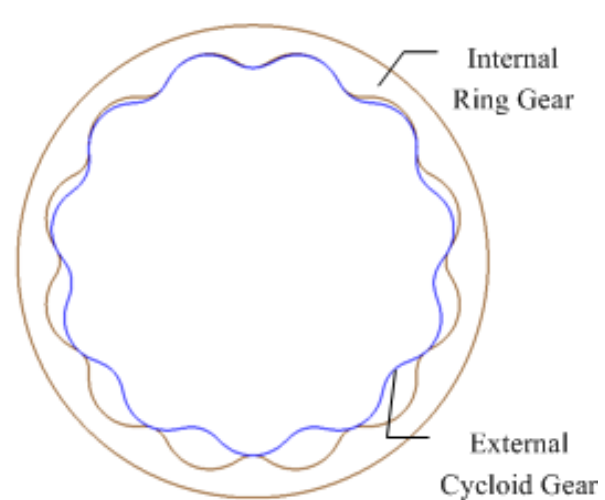

Fig. 2 Double-enveloping cycloid drive

The double-enveloping method and the generation process of the new conjugated tooth profile are as Fig. 3. According to the gear geometry theory ${ }^{(8)}$, the conjugated tooth profile can be obtained based on the original cutter tooth profile and the given relative enveloping motion. The tooth profile of external cycloid gear can be generated by using cylindrical pin roller as a cutter tool and the given relative enveloping motion. Further, if the cycloid gear is used the cutter tool profile, given the same relative enveloping motion, a new tooth profile which is called double-enveloping cycloid tooth profile in this paper can be generated. A new internal ring gear can be developed using the new double-enveloping cycloid tooth profile. And the conjugated gear pair of the double-enveloping cycloid drive is composed by the external cycloid gear and the new internal ring gear. The relative enveloping motion which generates external cycloid gear using pin rollers is called the first enveloping motion, and the motion which generates internal ring gear based on external cycloid gear is called the second enveloping motion.

The detailed mathematical derivation process of the new tooth profile is presented in another companion paper. In this paper the generation process is described summarily as the following. The coordinate systems are established according to the right-hand rule and shown in Fig. 4, where member 1 represents the internal ring gear and member 2 represents the planetary cycloid gear. The moving coordinate systems $O_{b} X_{1} Y_{1} Z_{1}$ and $O_{g} X_{2} Y_{2} Z_{2}$ are rigidly connected to the centers of the internal ring gear and the planetary cycloid gear, 
respectively. The fixed coordinate system $O X Y$ is connected to the center of the internal ring gear. In the initial position, the axes $X_{1}$ and $X$ are coincident, $X_{2}$ is parallel with $X$. The axes $Y_{1}, Y_{2}$ and $Y$ are coincident. The axes $Z, Z_{1}$ and $Z_{2}$ are parallel with each other. The radius of pin teeth distributed circle is $R_{z}$, and the radius of pin tooth is $r_{z}$. The tooth numbers of the internal ring gear and planetary cycloid gear are $z_{b}$ and $z_{g}$, and the gear center distance is $e$. The method in case of crank arm $O_{b} O_{g}$ being fixed is adopted to simplify the discussion.

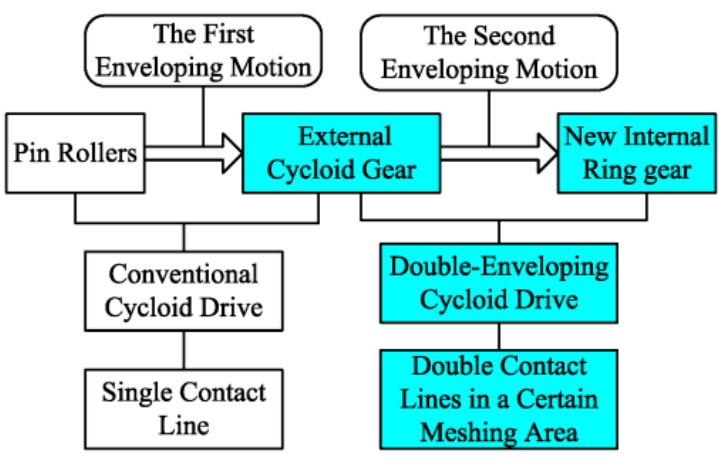

Fig. 3 Double-enveloping method

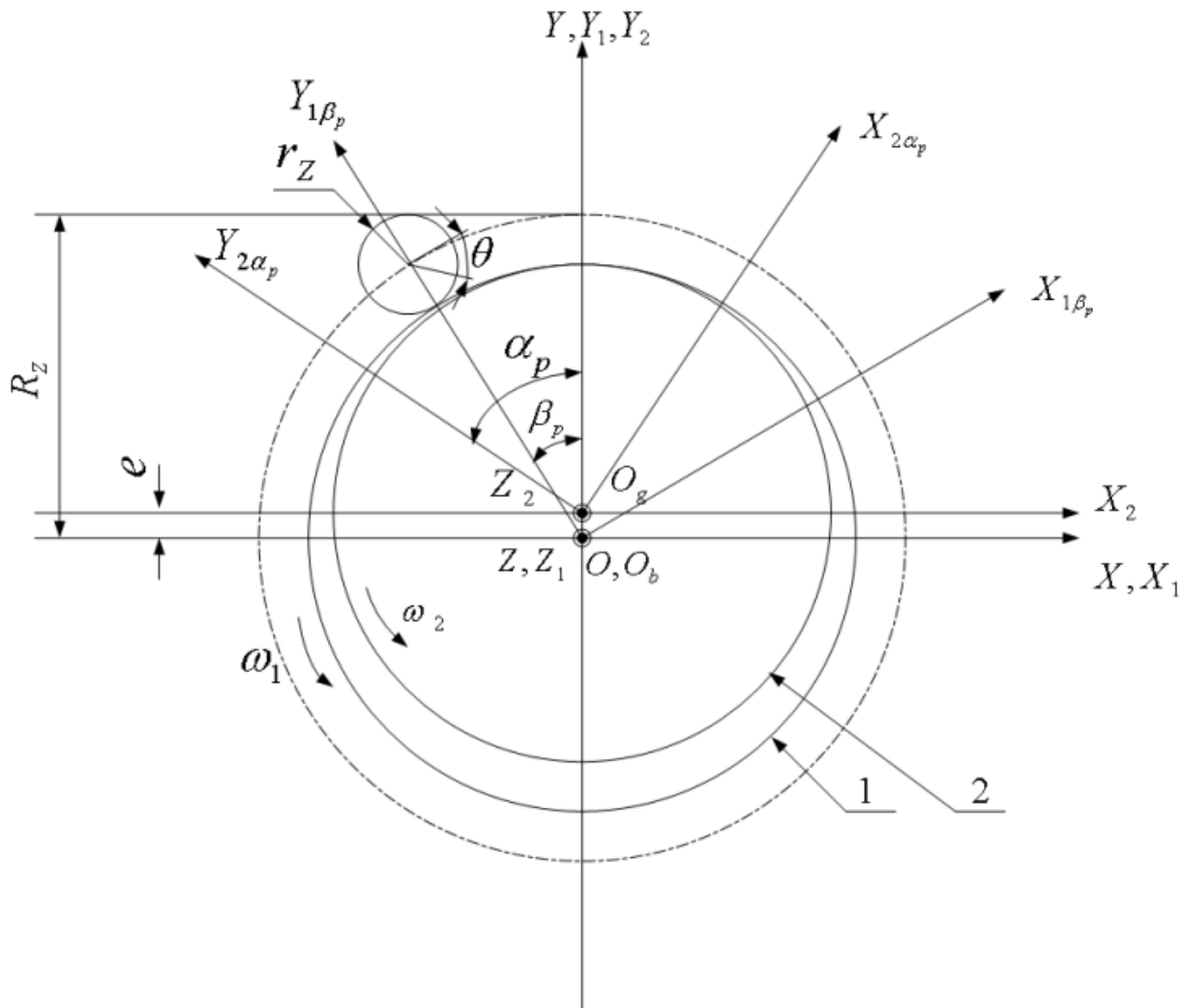

Fig. 4 Coordinate system

During the first enveloping motion, the pin roller is used as the cutting tool, when the internal ring gear rotates by angle $\beta_{1}$ with the speed $\omega_{1}$ about the axis $Z_{1}$ in counterclockwise direction, the coordinate system $O_{b} X_{1} Y_{1} Z_{1}$ which is connected to the internal wheel will rotate to the position $O_{b} X_{1 \beta 1} Y_{1 \beta 1} Z_{1}$, according to the relation of the relative motion, the cycloid gear will rotate by angle $\alpha_{1}$ with the speed $\omega_{2}$ about the axis $Z_{2}$ in the same direction, the coordinate system $O_{g} X_{2} Y_{2} Z_{2}$ which is connected to the cycloid gear will rotate to the position $O_{g} X_{2 \alpha 1} Y_{2 \alpha 1} Z_{2}$. During the second enveloping motion, the cycloid gear is used as the cutting tool, when the cycloid gear rotates by angle $\alpha_{2}$ with the speed $\omega_{2}$ about the axis $Z_{2}$ in counterclockwise direction, the coordinate 
system $O_{g} X_{2} Y_{2} Z_{2}$ which is connected to the connected to the cycloid gear will rotate to the position $O_{g} X_{2 \alpha 2} Y_{2 \alpha 2} Z_{2}$, according to the relation of the relative motion, the internal ring gear will rotate by angle $\beta_{2}$ with the speed $\omega_{1}$ about the axis $Z_{1}$ in the same direction, the coordinate system $O_{b} X_{1} Y_{1} Z_{1}$ which is connected to the internal ring gear will rotate to the position $O_{b} X_{1 \beta 2} Y_{1 \beta 2} Z_{1}$. In Fig. $4, p$ is subscript of $\alpha$ and $\beta$, where $p=1$ and $p=2$ represent the first and the second enveloping motion, respectively. The rotation speeds and rotation angles have the relationship as the following equation: $i=\omega_{2} / \omega_{1}=\alpha_{1} / \beta_{1}=$ $\alpha_{2} / \beta_{2}=z_{b} / z_{g}$, where $i$ represent the transmission ratio of the transform mechanism. And the meshing equation of the two enveloping motion can be obtained based on gear geometry theory.

The equation of the original cylindrical pin tooth profile $\Sigma^{(1)}$ can be described in the coordinate system $O_{b} X_{1} Y_{1} Z_{1}$ as Eq. (1).

$$
\left\{\begin{array}{l}
x_{1}=r_{z} \cos \theta \\
y_{1}=R_{z}+r_{z} \sin \theta \\
z_{1}=v
\end{array}\right.
$$

where $\theta$ and $v$ are the parameters of the tooth profile.

During the first enveloping motion, based on the original cylindrical pin and the given motion, the cycloid gear tooth profile $\boldsymbol{\Sigma}^{(2)}$ can be obtained by Eq. (2).

$$
\left\{\begin{array}{l}
\boldsymbol{\Sigma}^{(2)}=\mathbf{M}_{21} \boldsymbol{\Sigma}^{(1)} \\
\phi_{1}=\mathbf{n}_{1} \cdot \mathbf{v}_{1}^{(12)}=0
\end{array}\right.
$$

where $\mathbf{M}_{21}$ represents the transform matrix from coordinate $O_{b} X_{1} Y_{1} Z_{1}$ to $O_{g} X_{2} Y_{2} Z_{2} ; \phi_{1}$ represents the meshing equation of the first enveloping motion; $\mathbf{n}_{1}$ represents the unit normal vector to the pin roller tooth surface; $\mathbf{v}_{1}^{(12)}$ represents the relative velocity of the pin roller with respect to cycloid gear at the meshing point in coordinate system $O_{\mathrm{b}} X_{1} Y_{1} Z_{1}$.

By simplifying Eq. (2), the equation of the cycloid gear tooth profile $\Sigma^{(2)}$ can be described in the coordinate system $O_{\mathrm{g}} X_{2} Y_{2} Z_{2}$ as Eq. (3).

$$
\left\{\begin{array}{l}
x_{2}=R_{z} \sin \left[(i-1) \beta_{1}\right]+r_{z} \cos \left[\theta-(i-1) \beta_{1}\right]-e \sin \left(i \beta_{1}\right) \\
y_{2}=R_{z} \cos \left[(i-1) \beta_{1}\right]+r_{z} \sin \left[\theta-(i-1) \beta_{1}\right]-e \cos \left(i \beta_{1}\right) \\
z_{2}=v \\
\phi_{1}=\left[R_{z}(i-1) \cos \theta-i e \cos \left(\theta+\beta_{1}\right)\right] \omega_{1}=0
\end{array}\right.
$$

During the second enveloping motion, based on the cycloid gear and the same given motion, the double-enveloping cycloid tooth profile $\Sigma^{(1)^{\prime}}$ can be obtained by Eq. (4).

$$
\left\{\begin{array}{l}
\boldsymbol{\Sigma}^{(1)^{\prime}}=\mathbf{M}_{12} \boldsymbol{\Sigma}^{(2)} \\
\phi_{2}=\mathbf{n}_{2} \cdot \mathbf{v}_{2}^{(21)}=0
\end{array}\right.
$$

where $\mathbf{M}_{12}$ represents the transform matrix from coordinate $O_{g} X_{2} Y_{2} Z_{2}$ to $O_{b} X_{1} Y_{1} Z_{1} ; \phi_{2}$ represents the meshing equation of the second enveloping motion; $\mathbf{n}_{2}$ represents the unit normal vector to the cycloid gear tooth surface; $\mathbf{v}_{2}^{(21)}$ represents the relative velocity of the cycloid gear with respect to the internal ring gear at the meshing point in coordinate system $\mathrm{O}_{g} \mathrm{X}_{2} Y_{2} Z_{2}$.

By simplifying Eq. (4), the equation of the double-enveloping cycloid tooth profile $\Sigma^{(1)^{\prime}}$ can be described in the coordinate system $O_{b} X_{1} Y_{1} Z_{1}$ as Eq. (5).

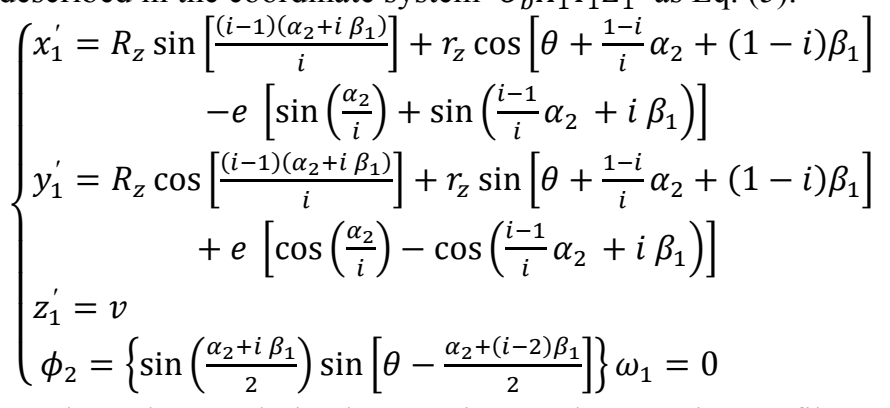

Based on the above derived equations, the tooth profile comparison of double-enveloping cycloid drive and conventional cycloid drive in terms of half tooth is as Fig. 5. The parameters of the investigated gear pair in this paper are as Table 1. 


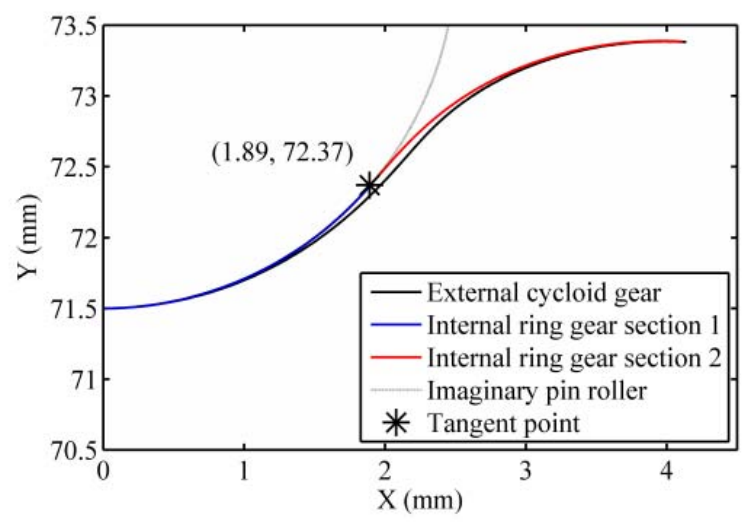

Fig. 5 Tooth profile comparison

Table 1 Gear parameters

\begin{tabular}{|c|c|c|}
\hline \multirow{2}{*}{$\begin{array}{c}\text { Teeth } \\
\text { number }\end{array}$} & \begin{tabular}{c} 
Internal ring gear \\
\cline { 2 - 3 } Radius
\end{tabular} & 56 \\
\hline \multirow{2}{*}{ gear } & Pin roller (mm) & 2.5 \\
\cline { 2 - 3 } & $\begin{array}{c}\text { Pin distribution } \\
\text { circle (mm) }\end{array}$ & 74 \\
\hline Distance of gear center (mm) & 1 \\
\hline Width of cycloid gear (mm) & 14.5 \\
\hline \multicolumn{2}{|c|}{ Elastic modulus (GPa) } & 206 \\
\hline \multicolumn{2}{|c|}{ Poisson ratio } & 0.3 \\
\hline
\end{tabular}

\subsection{Meshing characteristics}

From Fig. 5, each tooth of the new internal ring gear is composed by two sections: section 1 is the same as the cylindrical pin roller, section 2 is generated during the second enveloping motion. The contact lines at different crank shaft rotation angles are as Fig. 6. From Fig. 6, the new conjugated tooth profile has double contact lines in some certain meshing areas: the first contact line is between internal ring gear section 1 and the concave section of cycloid gear, the second contact line is between internal ring gear section 2 and the convex section of cycloid gear. During one rotation cycle of the crank shaft, the internal ring gear section 1 contacts twice with cycloid gear, whereas section 2 contact only once with the cycloid gear. As to the numerical example in this paper, for the tooth pair in Fig. 6, when the crank rotates from 0 to -0.71 , there are double contact lines; when the crank rotates from -0.71 to $-\pi$, there is only one contact line.

\section{Torsional stiffness calculation}

\subsection{Calculation model}

For double-enveloping cycloid drive, the motion is transmitted by the contact lines between the internal ring gear and the cycloid gear. Due to the elastic deformation, the contact line will become a small surface area in the actual transmission. For one conjugated tooth pair, there are double contact lines in a certain meshing area. In this paper, the calculation model is established based on Hertz theory using the model of conventional cycloid drive and spur gear pair as reference ${ }^{(6)(9)}$. Each contact line between one conjugated tooth pair is simplified as two cylindrical stiffness bodies, as Fig. 7. It is assumed that the length of the deformation area is $2 a$ and the width is $b$.

Based on Hertz formula, the length of the deformation area $a$ can be calculated by Eq. (6).

$$
a=\sqrt{\frac{4 F_{i}}{\pi b}\left(\frac{\frac{1-\mu_{1}^{2}}{E_{1}}+\frac{1-\mu_{2}^{2}}{E_{2}}}{\frac{1}{\rho_{i}}}\right)}
$$



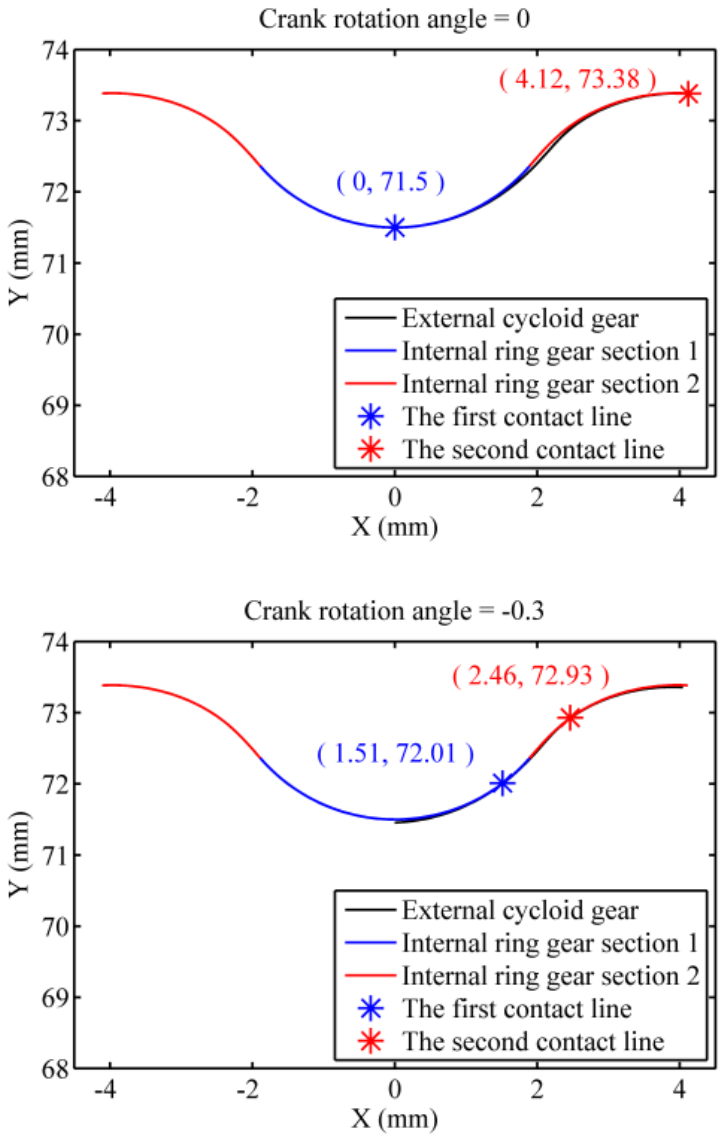

Crank rotation angle $=-0.71 \quad$ ( critical meshing position )
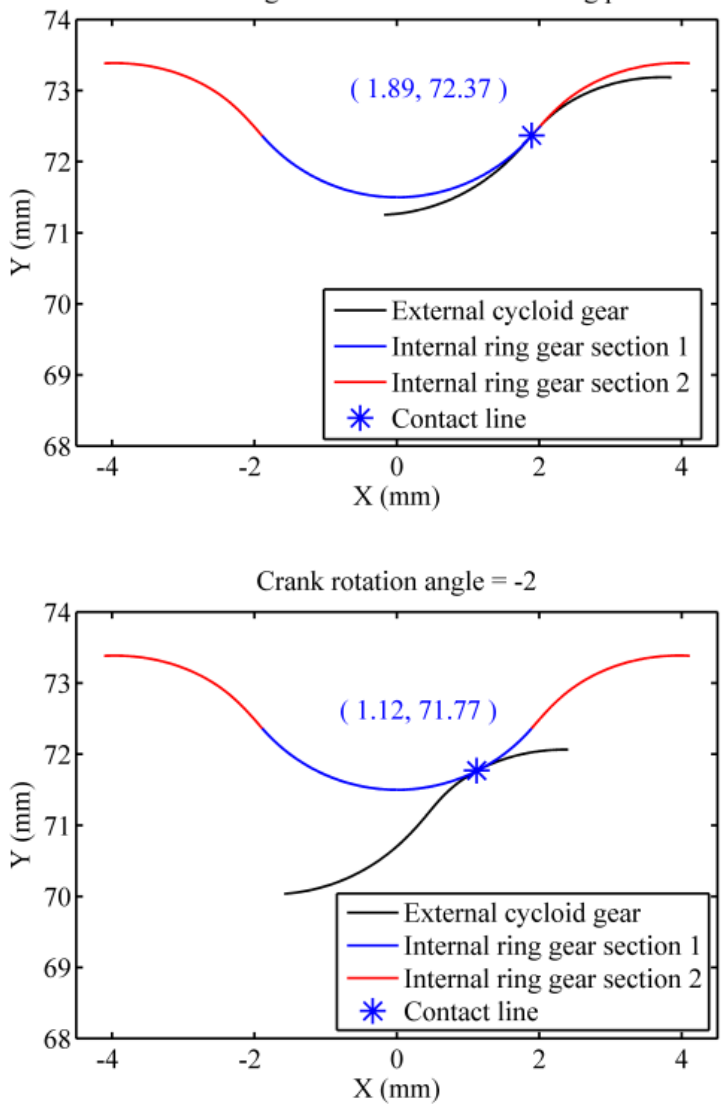

Fig. 6 Contact lines 
where $\rho_{i}$ is the compositive curvature radius between the internal ring gear and the cycloid gear; $E_{1}$ and $E_{2}$ are the elastic modulus of the internal ring gear and the cycloid gear, respectively; $\mu_{1}$ and $\mu_{2}$ are the Poisson ratio of the internal ring gear and the cycloid gear, respectively; $F_{i}$ is the meshing force.
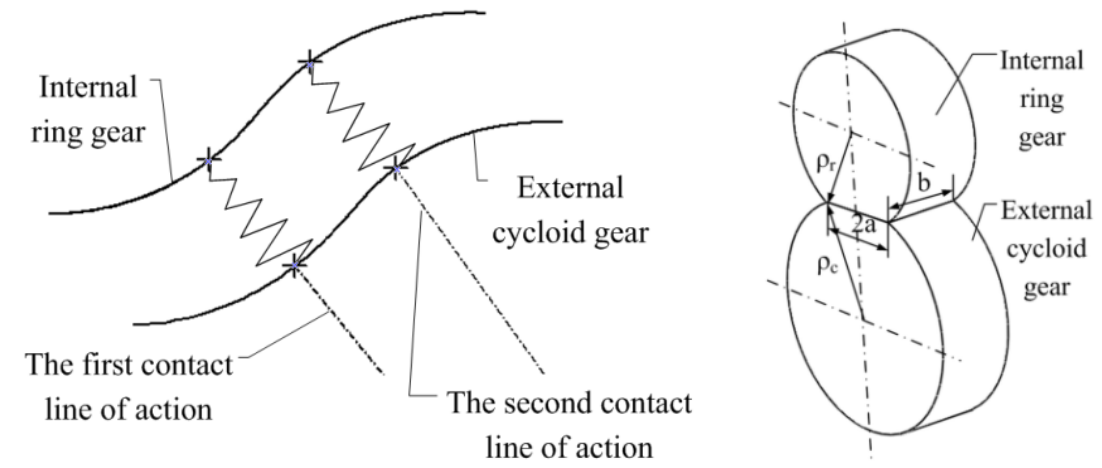

Fig. 7 Stiffness calculation model

In this paper, the material of the internal ring gear and the cycloid gear is assumed the same. That is $E_{1}=E_{2}=E$ and $\mu_{1}=\mu_{2}=\mu$. So Eq. (6) can be simplified as Eq. (7).

$$
a=\sqrt{\frac{8 F_{i} \rho_{i}\left(1-\mu^{2}\right)}{\pi b E}}
$$

The normal stiffness is defined as the ratio of the contact force between the two contacting surface to the deformation of the conjugated surface, and its direction is the same as the line of action. The main characteristic of conventional cycloid drive is that multi-tooth mesh simultaneously. At the same crank rotation position, the direction of the contact line is different for different meshing tooth pairs. So the normal stiffness direction is also different for different tooth pairs. As to double-enveloping cycloid drive, there are double contact lines in a certain meshing area besides the multi-tooth mesh simultaneously. The normal stiffness direction of the two contact lines for the same tooth pair is also different. In addition, the normal stiffness direction of the same tooth pair is also changed at different rotation positions. So the normal stiffness of the single tooth pair cannot be added simply for the whole gear mesh calculation. The torsional stiffness is defined as the ratio of the applied torque about the centroidal axis to the resulting angle, and it can be summed up directly for the whole gear mesh. In this paper, the normal stiffness of single tooth pair, the arm length, the torsional stiffness of single tooth pair and the whole gear mesh are calculated and investigated based on this model.

\subsection{Calculation of normal stiffness}

\subsubsection{Stiffness of single tooth of the internal ring gear}

From Fig. 7, the following equation can be obtained

$$
\left(\rho_{r}-t_{r}\right)^{2}+a^{2}=\rho_{r}^{2}
$$

where $t_{r}$ is the deformation of the internal ring gear tooth in the radial direction due to the meshing force; $\rho_{r}$ is the curvature of the internal ring gear.

Solving Eq. (8), the deformation $t_{r}$ can be calculated as Eq. (9).

$$
t_{r}=\frac{4 F_{i} \rho_{i}\left(1-\mu^{2}\right)}{\pi b E \rho_{r}}
$$

So the stiffness of the single tooth of the internal ring gear can be calculated by Eq. (10).

$$
K_{r i}=\frac{F_{i}}{t_{r}}=\frac{\pi b E \rho_{r}}{4\left(1-\mu^{2}\right) \rho_{i}}
$$

\subsubsection{Stiffness of single tooth of the external cycloid gear}

From Fig. 7, the following equation can be obtained

$$
\left(\rho_{c}-t_{c}\right)^{2}+a^{2}=\rho_{c}^{2}
$$

where $t_{c}$ is the deformation of the external cycloid gear tooth in the radial direction due to the meshing force; $\rho_{c}$ is the curvature of the external cycloid gear.

Solving Eq. (11), the deformation $t_{z}$ can be calculated as Eq. (12). 


$$
t_{c}=\frac{4 F_{i} \rho_{i}\left(1-\mu^{2}\right)}{\pi b E \rho_{c}}
$$

So the stiffness of the single tooth of the internal ring gear can be calculated by Eq. (13).

$$
K_{c i}=\frac{F_{i}}{t_{c}}=\frac{\pi b E \rho_{c}}{4\left(1-\mu^{2}\right) \rho_{i}}
$$

\subsubsection{Stiffness of single tooth pair}

The stiffness of single tooth pair between internal ring gear and external cycloid gear can be calculated by Eq. (14).

$$
K_{n i}=\frac{K_{r i} \cdot K_{c i}}{K_{r i}+K_{c i}}
$$

Substituting Eq. (10) and Eq. (13) into the Eq. (14), the following equation can be obtained

$$
K_{n i}=\frac{\pi b E \rho_{r} \rho_{c}}{4\left(1-\mu^{2}\right)\left(\rho_{r}+\rho_{c}\right) \rho_{i}}
$$

It is noted that in this calculation model the curvature values of the internal ring gear $\rho_{r}$ and the external cycloid gear $\rho_{c}$ in the formula are absolute values.

\subsection{Calculation of arm length}

The arm length of the meshing force is defined that the length between the center of the cycloid gear and the line of action, see Fig. 8.

The arm length of the first contact line can be calculated based on $\triangle P O_{g} A$ and $\triangle O_{b} P O_{1}$. In $\triangle P O_{g} A$, the arm length of the first contact line

$$
\begin{aligned}
& l_{1}=\overline{O_{g} A}=\overline{O_{g} P} \cdot \sin \angle O_{g} P A \\
& \sin \angle O_{g} P A=\sin \angle O_{p} P O_{1}=\sin \angle O_{1} P O_{b}
\end{aligned}
$$

In $\triangle O_{1} P O_{\mathrm{b}}$,

$$
\begin{aligned}
& \frac{\sin \angle O_{1} P O_{b}}{\overline{O_{1} O_{b}}}=\frac{\sin \angle O_{1} O_{b} P}{\overline{O_{1} P}} \\
& \overline{O_{1} P}=\sqrt{{\overline{O_{1} O_{b}}}^{2}+{\overline{O_{b} P}}^{2}-2 \cdot \overline{O_{1} O_{b}} \cdot \overline{O_{b} P} \cdot \cos \angle O_{1} O_{b} P}
\end{aligned}
$$

Substituting Eq. (17), Eq. (18) and Eq. (19) into Eq. (16), the equation of the arm length of the first contact line is obtained as

$$
l_{1}=\frac{\overline{o_{g} P} \cdot \sin \angle O_{1} O_{b} P}{\sqrt{1+\left(\frac{\overline{o_{b} P}}{\overline{o_{1} O_{b}}}\right)^{2}-2 \cdot \frac{\overline{o_{b} P}}{\overline{o_{1} O_{b}}} \cdot \cos \angle O_{1} O_{b} P}}
$$

where $\overline{O_{g} P}$ is the radius of the cycloid gear pitch circle, $\overline{O_{g} P}=e \cdot z_{g} ; \overline{O_{b} P}$ is the radius of the internal ring gear pitch circle, $\overline{O_{b} P}=e \cdot z_{b} ; \overline{O_{1} O_{b}}$ is the radius of pin teeth distributed circle, $\overline{O_{1} O_{b}}=R_{z} ; \angle O_{1} O_{b} P$ is the angle between the crank and the line which

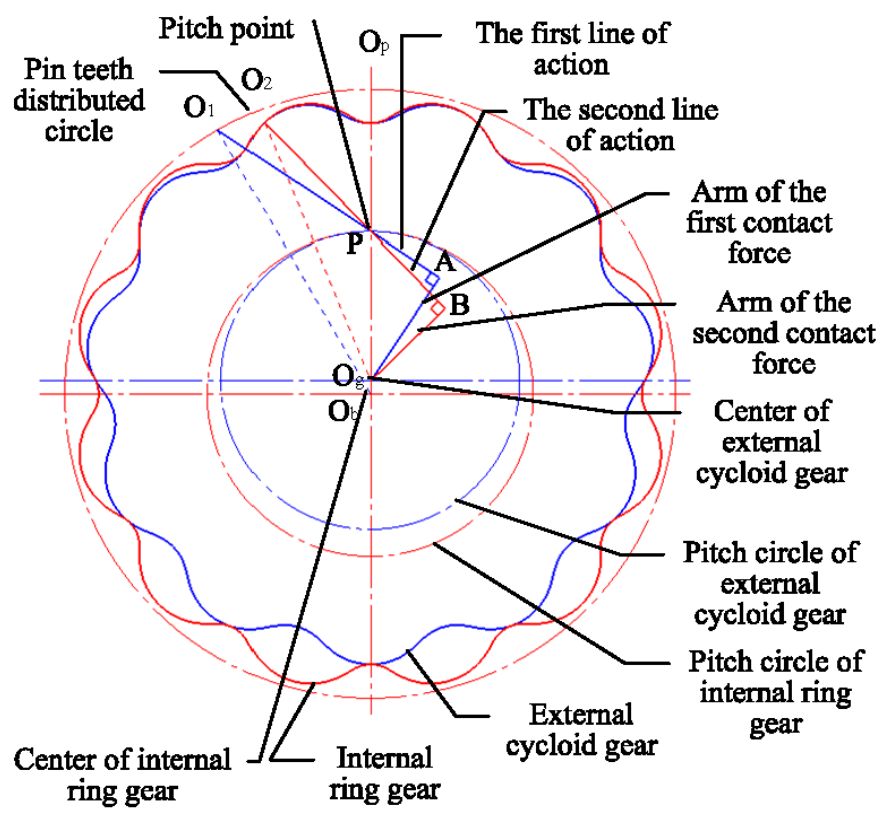

Fig. 8 Arm length calculation model 
through the internal ring gear center and the imaginary pin roller center.

The arm length of the second contact line can be calculated $\triangle P O_{g} B$ and $\triangle O_{g} P O_{2}$. In $\triangle P O_{g} B$, the arm length of the second contact line

$$
\begin{gathered}
l_{2}=\overline{O_{g} P} \cdot \sin \angle O_{g} P B \\
\sin \angle O_{g} P B=\sin \angle O_{p} P O_{2}=\sin \angle O_{2} P O_{g}
\end{gathered}
$$

In $\triangle O_{2} P O_{g}$

$$
\begin{gathered}
\frac{\sin \angle O_{2} P O_{g}}{\overline{O_{2} O_{g}}}=\frac{\sin \angle O_{2} O_{g} P}{\overline{O_{2} P}} \\
\overline{O_{2} P}=\sqrt{{\overline{O_{2} O_{g}}}^{2}+{\overline{O_{g} P}}^{2}-2 \cdot \overline{O_{2} O_{g}} \cdot \overline{O_{g} P} \cdot \cos \angle O_{2} O_{g} P}
\end{gathered}
$$

Substituting Eq. (22), Eq. (23) and Eq. (24) into Eq. (21), the equation of the arm length of the second contact line is obtained as

$$
l_{2}=\frac{\overline{O_{g} P} \cdot \sin \angle O_{2} O_{g} P}{\sqrt{1+\left(\frac{\overline{O_{g} P}}{\overline{O_{2} O_{g}}}\right)^{2}-2 \cdot \frac{\overline{O_{g} P}}{\overline{O_{2} O_{g}}} \cdot \cos \angle O_{2} O_{g} P}}
$$

where $\overline{\mathrm{O}_{2} \mathrm{O}_{g}}$ is the length between the second meshing point and the center of cycloid gear, $\overline{O_{2} O_{g}}=\sqrt{x_{2}^{1}+y_{2}^{1}} ; \angle O_{2} O_{g} P$ is the angle between the crank and the line which through the second meshing point and the center of the cycloid gear, $\angle O_{2} O_{g} P=\tan ^{-1}\left(\frac{x_{2}}{y_{2}}\right)$.

\subsection{Calculation of torsional stiffness for single tooth pair}

The torsional stiffness of single tooth pair can be calculated by Eq. (26).

$$
K_{t i}=K_{n i} \cdot l_{a}^{2}
$$

where $K_{t i}$ is the torsional stiffness for single tooth pair; a=1,2 represent the arm length of the first contact line and the second contact line, respectively.

\subsection{Calculation of torsional stiffness for whole gear mesh}

The main characteristic of the cycloid drive is that multi-tooth mesh simultaneously. Theoretically, it is half of the teeth number that takes part in meshing at the same time. However, in the actual transmission, the teeth that take part in meshing simultaneously will be reduced because of the error of the manufacturing and assembling. In this paper, for the convenience of the calculation and comparison, the meshing condition is assumed to the ideal condition that half of the teeth take part in meshing simultaneously.

The torsional stiffness of the whole gear mesh can be calculated by Eq. (27).

$$
K_{t}=\sum_{i=1}^{Z_{b} / 2} K_{t i}
$$

\section{Numerical calculation example}

\subsection{Calculation results}

The parameters of the numerical example in this paper are as Table1. The calculation of the internal ring gear curvature radius $\rho_{r}$, the external cycloid gear curvature $\rho_{c}$, and the compositive curvature radius $\rho_{i}$ have been presented in another companion paper, and the calculation result of compositive curvature radius $\rho_{i}$ is as Fig. 9.

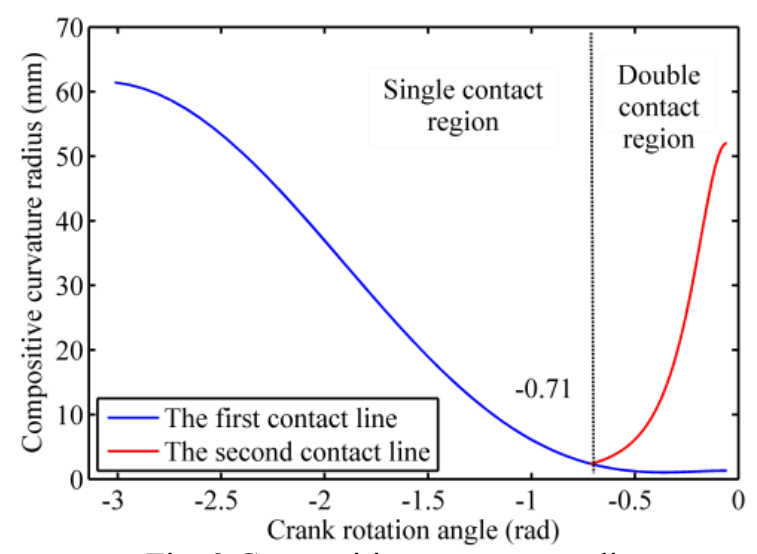

Fig. 9 Compositive curvature radius 
Substituting the gear parameters into the Eq. (15), Eq. (20) and Eq. (25), the normal stiffness of the single tooth pair and the arm length are calculated as Fig. 10 and Fig. 11.

Based on the calculation results of the normal stiffness and the arm length, the torsional stiffness of single tooth pair can be calculated by Eq. (26) as Fig. 12.

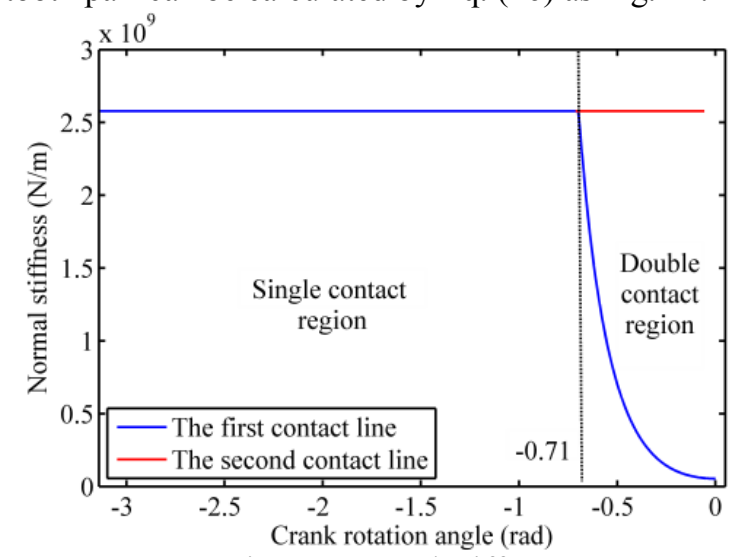

Fig. 10 Normal stiffness
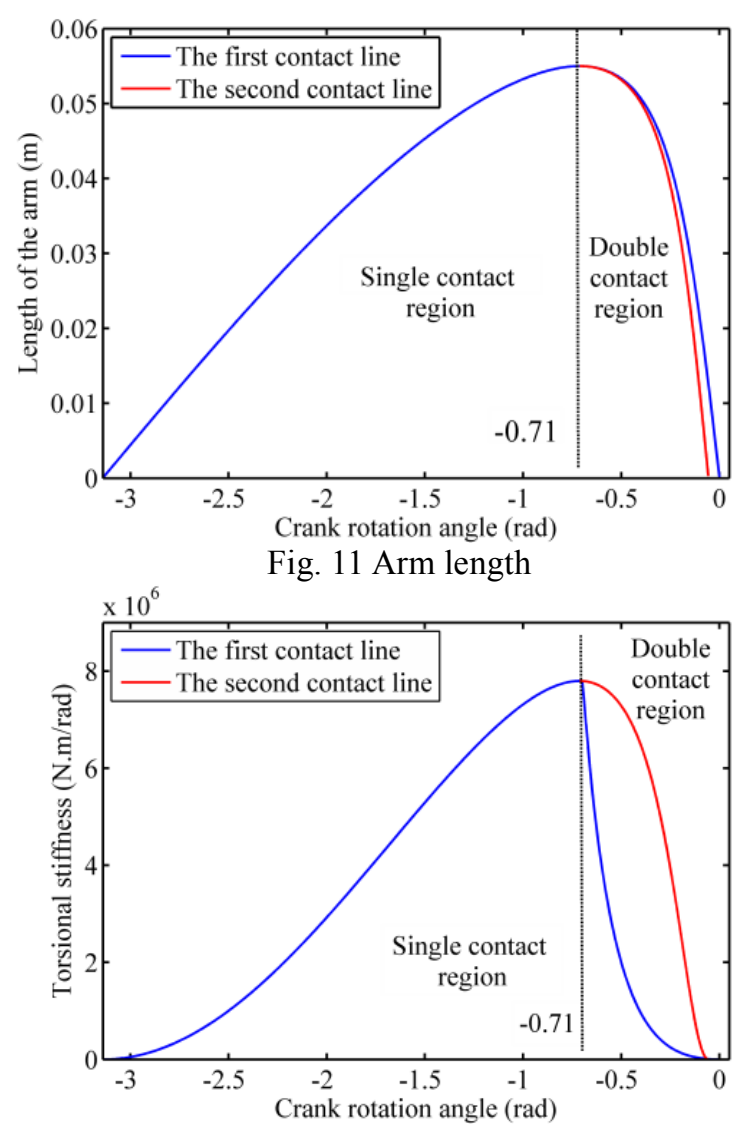

Fig. 12 Torsional stiffness

For double-enveloping cycloid drive, in the double contact region, the torsional stiffness of the two contact lines can be added directly to calculate torsional stiffness of the single tooth pair. The single tooth pair torisonal stiffness of the double-enveloping cycloid drive and the conventional cycloid drive during one crank rotation cycle are as Fig. 13 and Fig. 14.

Both of the double-enveloping cycloid drive and the conventional cycloid drive have a common characteristic: multi-tooth mesh simultaneously. The whole gear mesh torsional stiffness of double-enveloping cycloid and conventional cycloid drive during one crank rotation cycle are calculated by Eq. (27) as Fig. 15 and Fig. 16.

The whole gear mesh torsional stiffness comparison of double-enveloping cycloid drive 


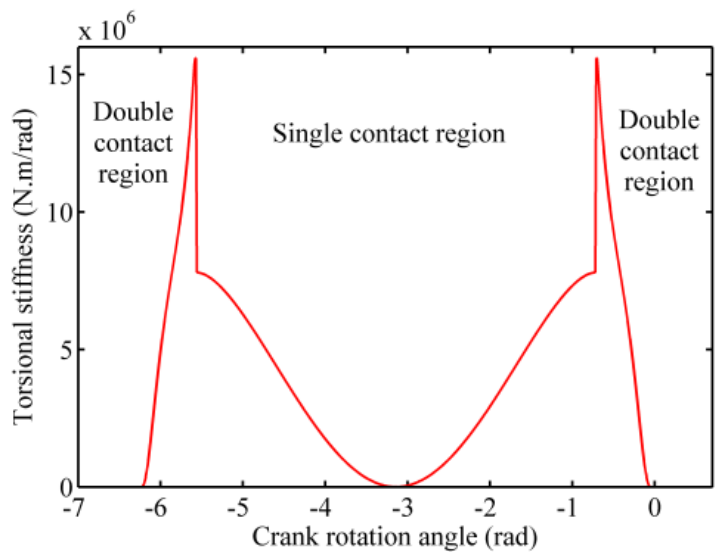

Fig. 13 Torsional stiffness of the double-enveloping cycloid drive: a single tooth engagement

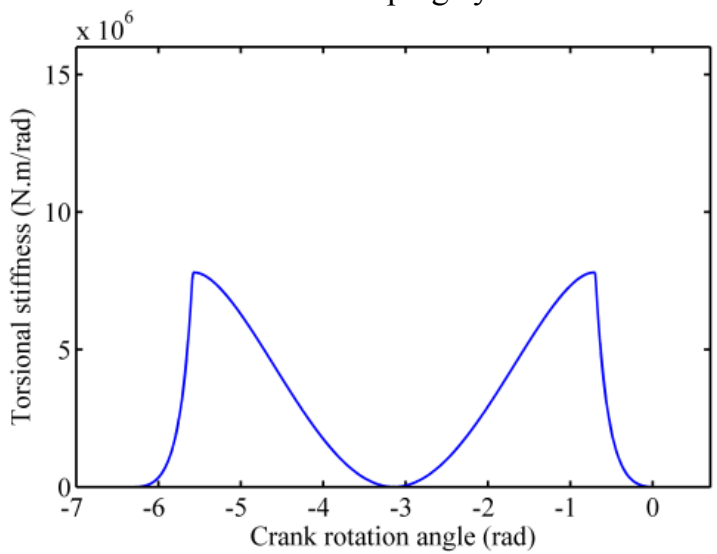

Fig. 14 Torsional stiffness of the conventional cycloid drive: a single tooth engagement

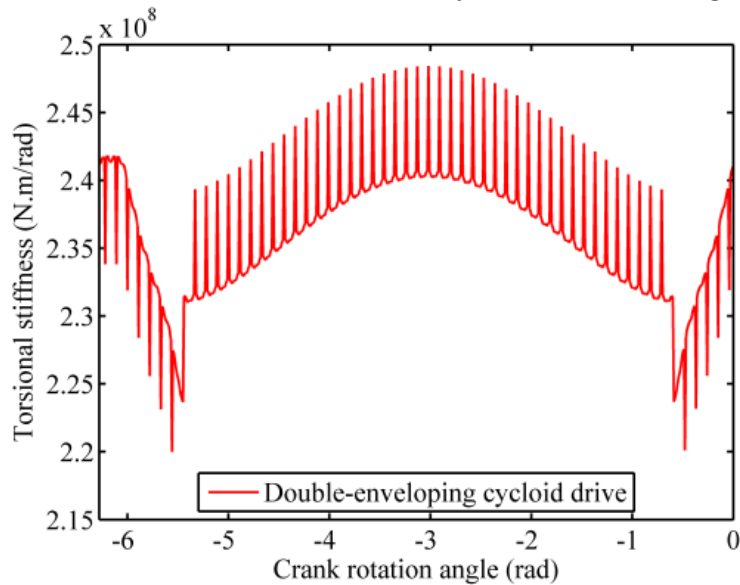

Fig. 15 Torsional stiffness of the double-enveloping cycloid drive: whole teeth engagement

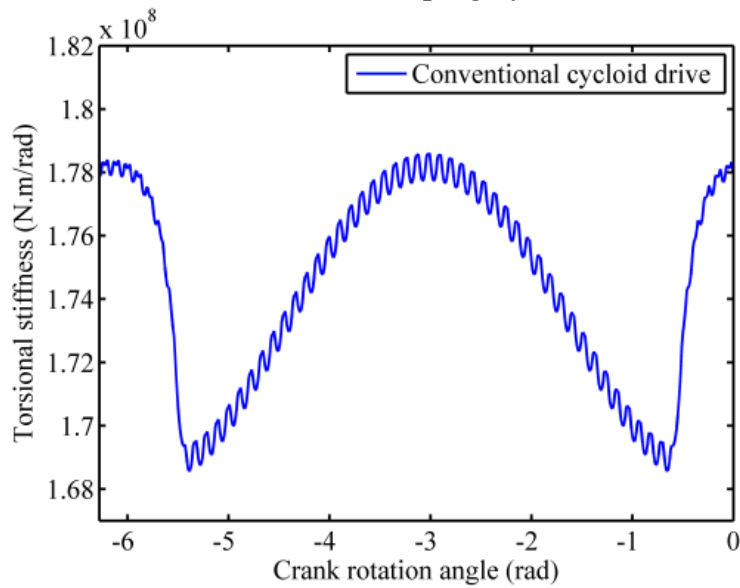

Fig. 16 Torsional stiffness of the conventional cycloid drive: whole teeth engagement 


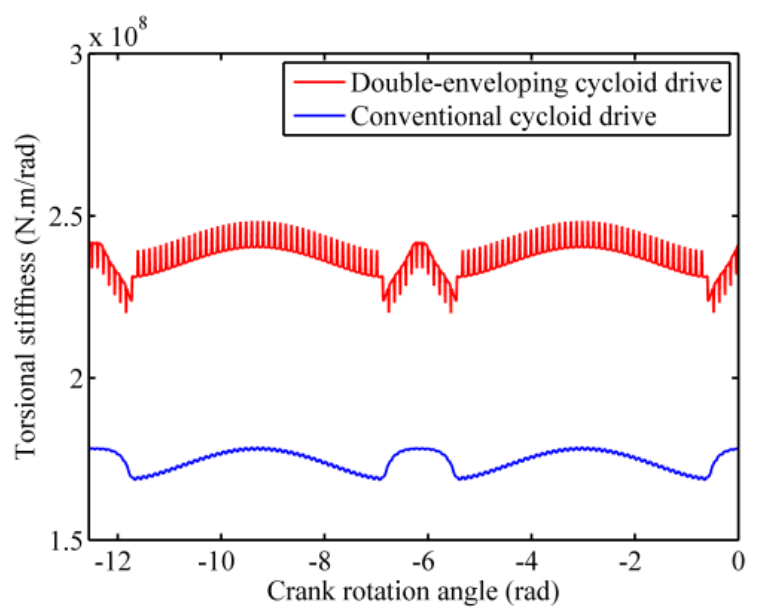

Fig. 17 Comparison of torsional stiffness

and conventional cycloid drive during two crank rotation cycles is as Fig. 17.

\subsection{Discussion}

From Fig. 10 and Fig. 12, for the first contact line, the normal stiffness is unchanged in the single contact region, and in the double contact region, it is changed gradually; the torsional stiffness is changed gradually in both of the two regions, and the maximal value exists at the critical meshing position of double contact and single contact region, also the change in the double contact region is drastic than that of the single contact region. For the second contact line, it is only exist in the double contact region, its normal stiffness is unchanged during the meshing process, and its torsional stiffness is changed smoothly than that of the first contact line. In addition, at the critical meshing position, the torsional stiffness curve connection for the second contact line is smoother than that of the first contact line.

From Fig. 13 and Fig. 14, the single tooth pair torsional stiffness of the double-enveloping cycloid drive is jump changed at the critical meshing position because the contact region changes from double contact to single contact line; whereas the change of the single tooth pair torsional stiffness of the conventional cycloid drive is consecutive.

From Fig. 15, Fig. 16, Fig. 17 and the statistics data, for conventional cycloid drive the average of the whole gear mesh torsional stiffness during one crank rotation cycle is $1.75 \times 10^{8} \mathrm{~N} . \mathrm{m} / \mathrm{rad}$, and the standard deviation is $3.19 \times 10^{6} \mathrm{~N} . \mathrm{m} / \mathrm{rad}$. Whereas for the double-enveloping cycloid drive the average torsional stiffness is $2.36 \times 10^{8} \mathrm{~N} . \mathrm{m} / \mathrm{rad}$, and the standard deviation is $4.64 \times 10^{6} \mathrm{~N} . \mathrm{m} / \mathrm{rad}$, which is $35 \%$ larger and $45 \%$ larger than that of conventional cycloid rive, respectively. So it can be concluded that the torsional stiffness of the double-enveloping cycloid drive is bigger but also larger fluctuations than that of conventional cycloid drive. The reason is that for each tooth pair of the double-enveloping cycloid drive, there are two contact lines in a certain meshing area, whereas in other meshing area there are only one contact line, so at the critical meshing position, the torsional stiffness has jump variation due to the change of number of the contact lines. As to the whole meshing gear pair, each tooth pair has a critical meshing position, also the critical meshing position for different tooth pair has phase difference from the viewpoint of the crank rotation angle, so the torsional stiffness of the whole meshing gear pair has many larger undulations. However, for conventional cycloid drive the ratio of the standard deviation to the average stiffness is $1.83 \%$. Whereas for double-enveloping cycloid drive this value is $1.97 \%$, which is almost the same with that of conventional cycloid drive. The reason is that the bigger average stiffness makes the spiky fluctuation the same relative contribution. From the view point of the excitation of stiffness variation for the gear pair vibration, the vibration of the double-enveloping cycloid drive will be almost the same with that of conventional cycloid drive. In addition, for the whole cycloid gear reducer, there are many other high stiffness mechanism elements, it is also possible that the tooth meshing 
stiffness has little influence on the output rotation vibration of the gear reducer. And the tooth meshing stiffness influence on the output rotation vibration for double-enveloping cycloid drive will be researched further in a following paper.

\section{Conclusion}

From the calculation results and discussion, the following conclusions can be made:

(1) For the two contact lines of the double-enveloping cycloid drive, the torsional stiffness characteristic of the second contact line is superior to that of the first contact line.

(2) For the same gear parameters, the torsional stiffness of the double-enveloping cycloid drive is larger than that of the conventional cycloid drive.

(3) The single tooth pair torsional stiffness of the double-enveloping cycloid drive has jump variation at the critical meshing position of double contact and single contact.

(4) The whole gear mesh torsional stiffness of the double-enveloping cycloid has many larger undulations. The vibration of the double-enveloping cycloid drive will be almost the same with that of the conventional cycloid drive from the viewpoint of stiffness variation excitation. The reason is that although the whole gear mesh torsional stiffness of the double-enveloping cycloid drive has many larger spiky fluctuations, the big average stiffness makes it the same relative contribution.

\section{Acknowledgment}

The authors are grateful to the China Scholarship Council (CSC). Part of the work was under the support of the scholarship No.2009605049 of the State Scholarship Fund.

\section{References}

(1) Chen, B.K., Fang, T.T., Li, C.Y. and Wang, S.Y., Gear Geometry of Cycloid Drives, Science in China Series E: Technological Sciences, Vol.51, No.5 (2008), pp. 598-610.

(2) Sakai, T. and Maki, M., An Investigation on Secondary Action on Skew Gears, Transactions of the ASME, Journal of Engineering for Industry, (1974), pp. 25-32.

(3) Mohan, L.V. and Shunmugam, M.S., Geometrical Aspects of Double Enveloping Worm Gear Drive, Mechanism and Machine Theory, Vol.44, No.11 (2009), pp. 2053-2065.

(4) Shi, W.K., Qin, D.T. and Xu, W.J, Meshing Control of the Double-Enveloping Hourglass Worm Gearing Under the Conditions of Existing the Errors and the Load, Mechanism and Machine Theory, Vol.39, No.1 (2004), pp. 61-74.

(5) Ratanasumawong, C., Matsumura, S. and Houjoh, H., Inspection of Toooth Surface Geometry by Means of Vibration Measurement, JSME International Journal Series C, Vol.48,No.4 (2005), pp.704-714.

(6) Zhang, D., Wang, G. and Huang T., Dynamic Formulation of RV Reducer and Analysis of Structural Parameters (in Chinese), Chinese Journal of Mechanical Engineering (ISSN:0577-6686), Vol.37, No.1 (2001), pp. 66-74.

(7) Chen, B.K., "Secondary Envelop Cycloid Planetary Transmission Apparatus", Chinese Patent Disclosure ZL200610054263.6 (2008)

(8) Litivin, F.L. and Fuentes, A., Gear Geometry and Applied Theory, (2004), p.97-152, Cambridge University Press.

(9) Ogawa, Y., Matsumura, S., Houjoh, H., SATO, T. and Umezawa, K., Rotation Vibration of a Spur Gear pair Considering Tooth Helix Deviation, JSME International Journal Series C., Vol.43, No.2 (2000), pp.423-431. 